\title{
PULSED POWER SYSTEM OF LINEAR INDUCTION ACCELERATOR FOR FNAL NEUTRINO FACTORY
}

\author{
V. Kazacha, A. Sidorov, JINR, Dubna, Moscow region, Russia \\ I. Terechkine, FNAL, Batavia, IL 60510, USA
}

\begin{abstract}
In the year 2000, a feasibility study of a Neutrino Factory (NF) was conducted at FNAL. As part of NF drift channel, phase rotation scheme involving long-pulse accelerating system was suggested. This system should provide voltage pulse that would correct charged particles energy spread. To implement this approach, pulsed power system has been suggested that allowed forming a bipolar accelerating voltage pulse with predefined pulse shape. This report summarizes main features of the system that is to drive an accelerating structure similar to that of a linear induction accelerator (LIA).
\end{abstract}

\section{INTRODUCTION}

The draft parameters of the FNAL Neutrino Factory (NF) were presented in [1]. A possibility to use an induction linac (LIA) after the target and decay channel for phase rotation of the muon beam was further investigated in [2]. Before the muon beam goes into the Phase Rotation Channel (PRC), the particle energy spreads from $170 \mathrm{MeV}$ up to $520 \mathrm{MeV}$. This energy spread is too high for the NF accelerating channel, and it is necessary to reduce it by adjusting the muon beam differential energy and cooling. To allow the differential energy adjustment, the particles are sent through a drift channel to develop a correlation between their energy and position. Then the differential acceleration can be used in a manner that reduces the energy spread. The energy-time correlation calculated in [2] was used to define correcting accelerating voltage pulse shape that improves energy spectrum of muon beam. It is important that the accelerating voltage closely follows the theoretical correcting energy curve.

Because of a very large emittance of the beam coming from the target, a large aperture beam channel accompanied by a strong magnetic field is needed. The bore radius of the inductor cores must be more than $40 \mathrm{~cm}$ with the magnetic field of about 3.0 T.

Duration of the correcting voltage pulse is changing while particles drift in the LIA channel providing additional mass separation. The starting pulse length of $150 \mathrm{~ns}$ was accepted for this study with the maximal correcting energy swing of $200 \mathrm{MeV}$.

The LIA length must not significantly exceed $100 \mathrm{~m}$ to avoid excessive muon losses due to their decay. This results in a requirement of having the accelerating gradient of about $2 \mathrm{MV} / \mathrm{m}$.
The $15 \mathrm{~Hz}$ operation frequency and multiple pulse regime ( 4 pulses per $2 \mu \mathrm{sec}$ ) result in using "state-of-art" pulse power technique.

The total muon current does not exceed $100 \mathrm{~A}$. This means that the total system power is mainly defined by induction core power losses.

\section{GENERATOR SCHEME}

As it has been shown in [2] (see also Fig. 1 below), the correcting voltage pulse is quite nonlinear.

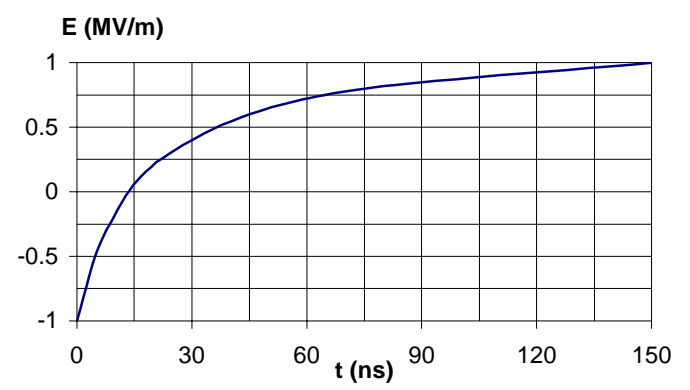

Figure 1: The calculated change of the voltage pulse shape on inductors versus time.

From the point of view of the phase rotation and subsequent cooling, it is not very important to have a certain average beam energy gain (or loss). It was shown though that some increase in the decelerating gradient would help to minimize the LIA energy consumption [3]. It means that on average the beam would loose its energy. In any case, the differential voltage must agree with the beam energy-time distribution found in [2]. Totally about $200 \mathrm{MV}$ of the accelerating voltage difference is required to equalize the muon beam energy using the phaserotating LIA. Since the "state-of-art" accelerating gradient in the LIA is about $1 \mathrm{MV} / \mathrm{m}$, we must consider the change of the voltage polarity to keep the LIA length in the range of about $100 \mathrm{~m}$.

The LIA pulsed power supply system is to provide a special regime of acceleration and to form the accelerating pulse shape that meets the requirements of the PRC. To adjust the working part of the pulse to the needed voltage level and to optimize the LIA parameters, a bias voltage pulse should be used. A conceptual scheme of the pulse generator is shown in Fig. 2.

The load of the generator is the LIA accelerating section that is made of ten induction cells connected in parallel. Every induction cell uses a tape-wound ferromagnetic core made of nanocrystalline alloy. Core 
cross-section is about $20 \mathrm{~cm}^{2}$. Because average core diameter is about $90 \mathrm{~cm}$, the volume of magnetic material per one core is about $5700 \mathrm{~cm}^{3}$. Although impedance of the induction cell is quite nonlinear due to the nonlinear nature of the power loss, for this study linear resistance was used to represent the load of the generator.

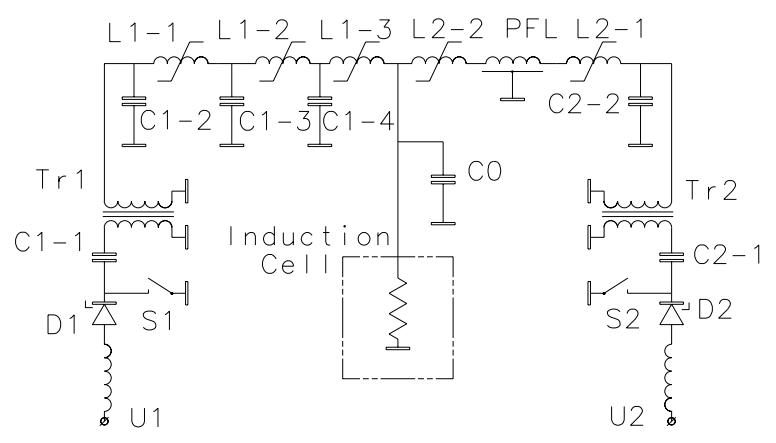

Figure 2: Conceptual scheme of the pulse generator.

There are two parts in the generator: the "charging" part, located on the left side of the picture in the Fig. 2, and the "forming" part on the right side of the picture. The "charging" part sets voltage of the capacitor $\mathrm{C} 0$ to the needed negative level. The "forming" part of the generator forms the output voltage that increases from the level of $-75 \mathrm{kV}$ up to $+25 \mathrm{kV}$. To produce needed differential voltage gain, 20 induction cells per each meter of LIA length are used, so that the first part of the beam sees negative voltage of $-1.5 \mathrm{MV}$ and the last part of the beam sees $+0.5 \mathrm{MV}$. The discharge of the capacitor $\mathrm{C} 0$ and pulse forming line (PFL) form the working part of the voltage pulse applied to the induction cell. To ensure the necessary charge rate of the capacitor $\mathrm{C} 0$, a three-stage magnetic compression circuit is used. The energy is delivered to this compression circuit by the storage capacitor C1-1 via the pulse transformer Tr1. The PFL in the "forming" part of the generator is charged via the single-stage magnetic compression circuit L2-1 $\div$ C2-2. The energy for this part of the circuit is stored in $\mathrm{C} 2-1$, and $\operatorname{Tr} 2$ is used to get the needed PFL voltage.

Figures 3 to 5 show voltage pulse diagrams of the generator. During the time interval from 0 till $60 \mathrm{~ns}$ (see Fig. 5) the "charging" part of the generator charges the capacitor $\mathrm{C} 0$ to $-95 \mathrm{kV}$. Starting at $60 \mathrm{~ns}$, a natural discharge of the capacitor $\mathrm{C} 0$ occurs through the induction cell. Starting this moment, the magnetic switch L1-3 magnetizes increasing its resistance to the positive voltage coming from PFL through the reactor L2-2. Staring at $67 \mathrm{~ns}$, the reactor L2-2 opens and the PFL begins to recharge the capacitor $\mathrm{C} 0$. At the same moment, the capacitor $\mathrm{C} 0$ continues to discharge through the induction cell. During this time interval (from $67 \mathrm{~ns}$ till $111 \mathrm{~ns}$ ) the magnetic switch L1-3 continues to magnetize and its resistance increases to the extent that allows to exclude the energy transfer from the PFL to the capacitor C1-4 in the positive part of the voltage pulse. The sufficiently large resistance of the magnetic switch L1-3 is reached if the negative volt-second area (from $60 \mathrm{~ns}$ till
$111 \mathrm{~ns})$ is, at least, equal to the area under the positive voltage pulse part (from $111 \mathrm{~ns}$ till $217 \mathrm{~ns}$ ). As calculations have shown, shortening the duration of the natural discharge of the capacitor $\mathrm{C} 0$, can reduce the "charging" voltage level. This duration (and the output voltage level) becomes lower when the asymmetrical voltage pulse to feed the induction cell is used with voltage shifted to the negative part of the voltage scale.

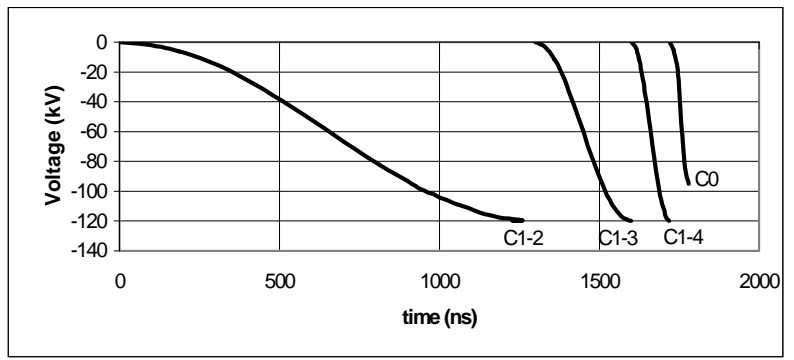

Figure 3: Voltage pulses for the "charging" part of the generator.

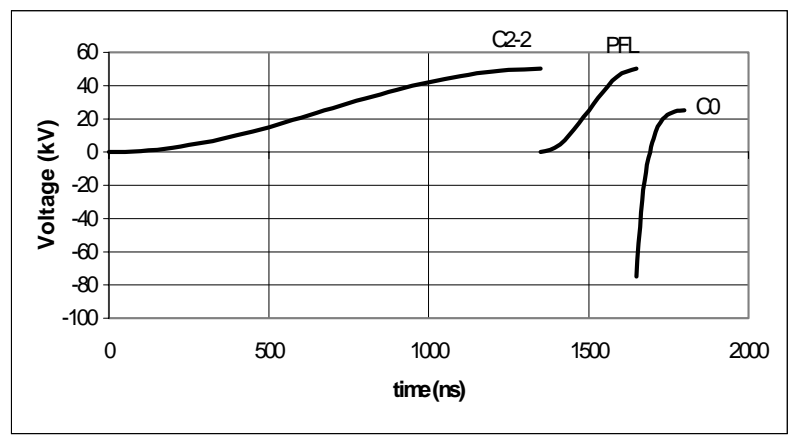

Figure 4: Voltage pulses for the "forming" part of the generator.

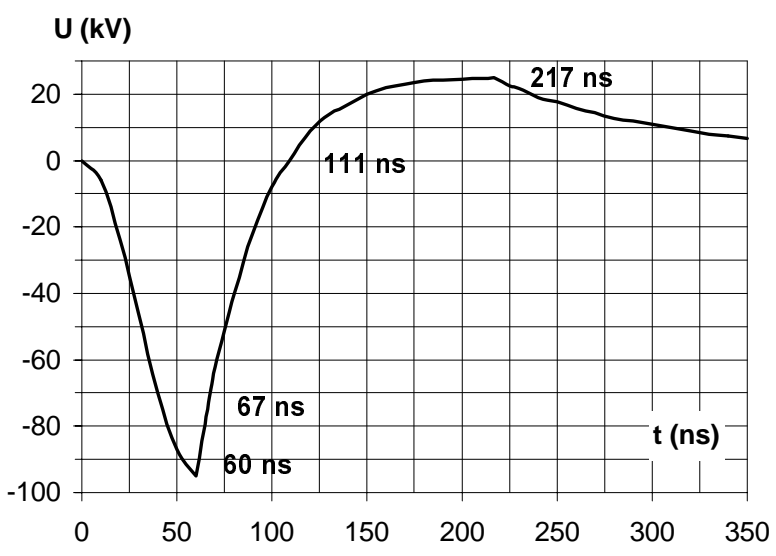

Figure 5: Output voltage pulse applied to the inductor in the LIA section.

As the output voltage pulse diagram (Fig. 5) shows, during the active part of the pulse that starts at $67 \mathrm{~ns}$ and is used for the phase rotation, induction cell voltage changes from $-75 \mathrm{kV}$ up to $+25 \mathrm{kV}$. The time duration of the negative and positive parts of the voltage pulse (not only of the active part of the pulse) is chosen to make their volt-second areas equal to each other. As a result, the 
magnetic recovery of the induction cell core is reached automatically, and when one voltage pulse is over, the system is ready to accept the next one. The next pulse can start immediately after the previous one ends.

Changing the following three parameters can change the shape of the output voltage pulse: the capacitance value of the $\mathrm{C} 0$, the inductance value of the reactor $\mathrm{L} 2-2$, and the PFL charging voltage. This option is very important: because particle energy distribution in one bunch changes while it is moving along the LIA, correcting voltage shape must also be changed.

Impedance of the described circuit cannot be made arbitrarily small. Nevertheless, it appears possible to use one generator to feed 10 inductors (0.5-m length) in parallel. This sets a natural limit to the LIA accelerating section length and its possible increment as $0.5 \mathrm{~m}$. The final choice of this length must be done based on other requirements of mechanical, magnetic or other nature.

The four-pulse regime can be realized by using the generator scheme shown in Fig. 6. Both "charging" and "forming" parts of the generator contain four primary circuits. Each primary circuit acts once during $2-\mu$ s time span so that four voltage pulses are generated. Each primary circuit in the "charging" part of the generator is separated from the rest three circuits by nonlinear reactors L1-1 $\div$ L1-4 (magnetic switches). In order to avoid energy transfer into unused primaries, the reactors' volt-second areas were chosen to be by a factor of 3 greater than the volt-second areas of the magnetic switches L1-5 and L1-6 taken together. The same logic was used to choose parameters of the reactors L2-1 $\div$ L2-4 of the "forming" part of the generator. An appropriate demagnetizing system must be used to set all magnetic switches into the initial position before the next set of four pulses can be generated. The $15 \mathrm{~Hz}$ repetition rate provides plenty of time to make it.

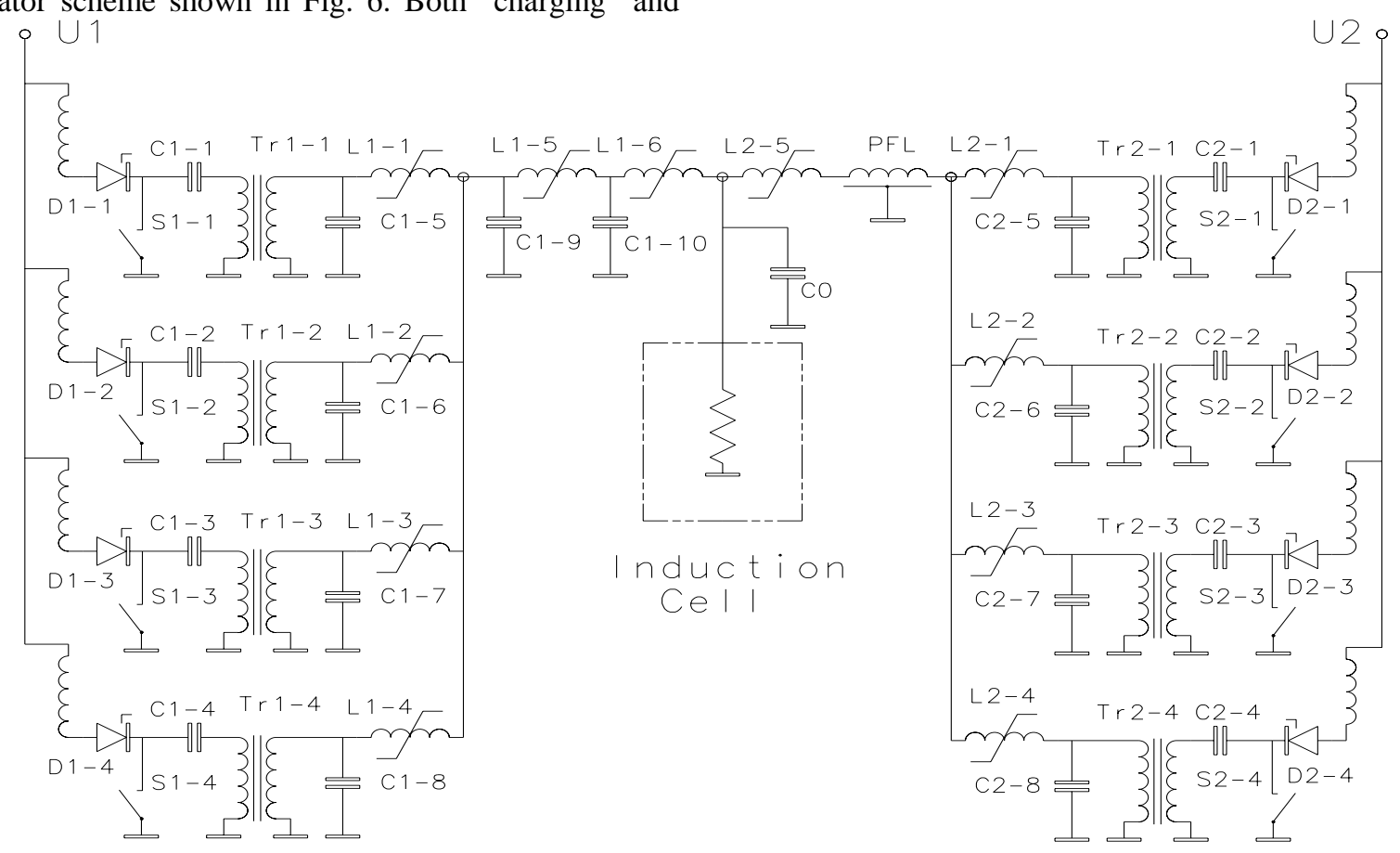

Figure 6: Principal scheme of the LIA pulse generator.

\section{CONCLUSIONS}

The pulsed power system for the LIA of the FNAL version of Neutrino Factory has been developed that allows forming the accelerating voltage pulse with the required shape and timing pattern.

Generation of the repetitive sequence of several pulses does not require any additional means to recover the initial state in the induction cores.

The next step of the LIA development should include a thorough simulation of the pulsed power circuits that takes into account nonlinear induction cell impedance, stray inductance and capacitance of the constructive elements of the accelerating section.

\section{REFERENCES}

[1] R.B. Palmer, "Neutrino Factory Draft Parameters", Muon Collider Collaboration Note \#46, Sept. 1999.

[2] V. Balbekov, N.Holtkamp, "Phase Rotation of Muons by Induction Linac", Muon Collider Collaboration Note \#59, Oct. 1999.

[3] V. Kazacha, A. Sidorov, Yu. Terechkine, "Generation of Multiple Bipolar Voltage Pulse for a Phase Rotation LIA", presented at PPPC-2001, Las-Vegas, June 18 - 22, 2001. 